\title{
The Effect of Adding Carbon Nitride Nanometer Material on The Properties of Epoxy Resin Project
}

\author{
Xing Wang ${ }^{1,2}$, Wenliang Sun ${ }^{1}$ and Min Guo ${ }^{2, a}$ \\ ${ }^{1}$ School of Economics and Management. Hebei University of Technology, 17 Fukang Road,Nankai District, Tianjin 300191, China \\ ${ }^{2}$ Tianjin Academy of Environmental Sciences, 17 Fukang Road,Nankai District, Tianjin 300191,China
}

\begin{abstract}
Boron nitride $(\mathrm{BN})$ nanometer material was adding as filler to epoxy resin to obtain a high thermal conductive and excellent mechanical properties material. The fillers, which include globular BN, flake BN and fibrous BN, were treated with silane coupling agent and acid to establish a strong affinity with the surrounding matrices. As a result, the thermal conductivity and mechanical properties of the composites were enhanced at a relatively appropriate volume fraction of BN. At a given volume fraction, fillers treated with silane coupling agent provide composites with lower thermal conductivity and higher tensile strength compared with the ones treated with acid. With the increase of the volume fraction of fillers, the thermal conductivity and the tensile strength of the composites show the tendency of increasing, but the tensile yield stress of composites exhibit significant reduction.
\end{abstract}

\section{Introduction}

Epoxy resin as the polymer matrix were used most often with reinforcing fibers for advanced composite applications [1]. With the development of epoxy composite material with both high thermal conductivity and excellent mechanical properties in various areas of weight-sensitive aerospace industry, marine, armor, automobile, railway and sporting goods industries, filling inorganic particles of nanometer size with high thermal conductivity and excellent mechanical performance has become a attractive and effective method to strengthen the properties of epoxy resins and meet the higher requirements. Because micro-scale fillers have successfully been synthesized with epoxy resin, BN as a novel nano-scale filler material, which include globular $\mathrm{BN}$, flake $\mathrm{BN}$ and fibrous $\mathrm{BN}$, is now being tested to produce high performance composite structures with enhanced properties [1-14]. Recently, boron nitride (BN) based nanofillers such as BN nanoplatelets, BN nanosheets (BNNSs) and BN nanotubes (BNNTs) have attracted increased attention due to its low density, high thermal conductivity, electrical insulation, superb oxidation resistance, passivity to reactions with acids and melts, low coefficient of friction and their potential application in preparing polymer composites for thermal dissipation [15-16].

So far, there have been two approaches developed for nanofiller surface functionalization: covalent and noncovalent methods [2]. The former involves the grafting of reactive modifiers onto the surface of the nanofillers by covalent bonds, while the later is applying the physical interaction between the fillers and modifiers such as van der Walls attractions, electrostatic interactions and hydrogen bonding [17-18].

The purpose of this paper is to show the effect of BN on the thermal and mechanical properties of epoxy [1]. Epoxy resin is chosen as the matrix because of its extremely wide application in aerospace, automotive and electronic industries [2,19]. Zhi et al. prepared a series of polymer nanocomposites with excellent thermal properties by using BNNSs and BNNTs as fillers [20-21]. Previous reports show that various methods have been developed to functionalize BNNSs and BNNTs in order to achieve better processability of their composites . We cured a series of epoxy composites by using different surface functionalization of BN fillers, and the method of the functionalization belongs to covalent functionalization. The BN fillers were firstly treated with silane coupling agent and acid to introduce amine groups and hydroxy groups. The strong covalent bonding formation produced a strong interface between fillers and matrix [2].

\section{Experimental}

\subsection{Materials}

The $\mathrm{BN}$ powers (globular $\mathrm{BN}$, flake $\mathrm{BN}$ and fibrous BN) with an average of $30 \mu \mathrm{m}$, concentrated sulfuric acid and nitric acid, ethanol, deionized water, hydrochloric acid, centrifuge, electronic stir bar, disposable straws, beaker, evaporating dish, tweezers, disposable gloves, electronic scale, $50 \mathrm{ml}$ beaker, flake BN, thermometer, electronic mixer, epoxy resin and curing agent.

\footnotetext{
a Corresponding author: 251939587@qq.com
} 


\subsection{Treatment with silane coupling agent}

Ethanol and deionized water was mixed at the mass radio of 9:1 uniformly (45g of ethanol and $5 \mathrm{~g}$ of deionized water), 3 drops of hydrochloric acid was added into the mixture to adjust ph value to 4 , the silane coupling agent $\mathrm{KH}-550$ at the mass fraction of $1.75 \%$ was added into the aqueous solution of hydrochloric acid $(7 \mathrm{~g})$, the aqueous solution was placed $3 \mathrm{~h}$ at room temperature to hydrolysis, then the powers of globular $\mathrm{BN}$ were mixed into the solution at the mass radio of 2:7 with $\mathrm{KH}-550$, the mixture was stirred $2 \mathrm{~h}$ at room temperature and was centrifugal, then it was cleaned with alcohol solution several times until the solution was neutral, finally, the solution obtained was placed in the evaporating dish to dry at the temperature of $50^{\circ} \mathrm{C}$ with 3 min and took out, then the solid obtained was ground into a fine powder and collectted to use. The powers of flake $\mathrm{BN}$ and fibrous $\mathrm{BN}$ were processed in the same way.

\subsection{Treatment with acid}

The concentrated sulfuric acid and nitric acid was took at volume radio of $3: 1(30 \mathrm{ml}$ of concentrated sulfuric acid and $10 \mathrm{ml}$ of concentrated nitric acid), $1 \mathrm{~g}$ of powers of globular $\mathrm{BN}$ were added, then the mixture was stirred $3 \mathrm{~h}$ at the temperature of $50^{\circ} \mathrm{C}$ with water bath heating and was centrifugal, then it was cleaned with deionized water several times until the solution was neutral, the solution obtained was placed in the evaporating dish to dry at the temperature of $50^{\circ} \mathrm{C}$ with 6 min and took out, then the solid obtained was ground into a fine powder and collectted to use. The powers of flake $\mathrm{BN}$ and fibrous $\mathrm{BN}$ were processed in the same way.

\subsection{Adding 1\%, $2 \%, 3 \%$ BN powers to EP}

$20 \mathrm{ml}$ of epoxy resin and $0.3 \mathrm{~g}$ of powers of globular $\mathrm{BN}$ was added into the breaker respectively, the mixture was stirred $2 \mathrm{~h}$ at the temperature of $50^{\circ} \mathrm{C}$, then the $10 \mathrm{ml}$ of curing agent was added into the breaker, the mixture was stirred $0.5 \mathrm{~h}$ at the temperature of $50^{\circ} \mathrm{C}$ with water bath heating, finally the mixture was encapsulated and cooled at room temperature. According to the methods above, $0.6 \mathrm{~g}$ and $0.9 \mathrm{~g}$ of powers of globular BN were added into epoxy resin respectively. The powers of flake $\mathrm{BN}$ and fibrous BN were added into epoxy resin at the same volume radio in the same way.

\subsection{The preparation of neat sample}

$20 \mathrm{ml}$ of epoxy resin was added into the breaker and the mixture was stirred $2 \mathrm{~h}$ at the temperature of $50^{\circ} \mathrm{C}$, then the $10 \mathrm{ml}$ of curing agent was added into the breaker, the mixture was stirred $0.5 \mathrm{~h}$ at the temperature of $50^{\circ} \mathrm{C}$ with water bath heating, finally the mixture was encapsulated and cooled at room temperature.

\subsection{Characterization}

Tensile tests were performed according to CMT-6104 under a two- end tensile configuration. The tests were conducted in a $10 \mathrm{KN}$ servo-hydraulic testing machine equipped with a Test Ware data acquisition system. The machine was run at a speed of $6 \mathrm{~mm} / \mathrm{min}$. All the tests were performed at room temperature. Test samples were cut and polished by using NHY-W (universal sample preparation instrument) and XFX (the dumbbell system prototype). Nine specimens from three different materials were prepared for tensile tests.

\section{Results and discussion}

\subsection{The data of tensile properties of composites and data analysis}

Typical stress-strain behavior from the tensile tests is shown in Fig.1, Fig.2, Fig3, Fig4. All specimens failed immediately after the tensile stress reached the maximum value. The stress-strain curves showed considerable nonlinearity before reaching the maximum stress, and obvious yield point. was found in the curves. Three specimens were tested for each condition.

Table 1. Mechanical properties of neat epoxy and epoxy/BN composites.

\begin{tabular}{|c|c|c|c|c|c|c|c|c|}
\hline & $\begin{array}{l}\text { Specinen } \\
\text { with } \\
(\mathbf{m m})\end{array}$ & $\begin{array}{l}\text { Specimen } \\
\text { ticklves } \\
(\mathbf{m m})\end{array}$ & $\begin{array}{l}\text { Modils } \\
\text { (MPa) }\end{array}$ & $\begin{array}{l}\text { dengation } \\
\text { athreak } \\
\text { (\%) }\end{array}$ & $\begin{array}{l}\text { Shrenght } \\
\text { (MPa) }\end{array}$ & $\begin{array}{c}\text { tend } \\
\text { fracture } \\
\text { shess } \\
(\mathrm{MPa}) \\
\end{array}$ & $\begin{array}{l}\text { tend } \\
\text { yetl } \\
\text { stues } \\
(\mathbf{M P a}) \\
\end{array}$ & $\begin{array}{l}\text { yeth } \\
\text { stain }\end{array}$ \\
\hline neat & 10.72 & 3.2 & 455.75 & 12.03 & 31.32 & 25.23 & 31.32 & 12.03 \\
\hline $\begin{array}{c}0.5 \% \\
\text { BN(Acid) }\end{array}$ & 10.76 & 3.09 & 459.24 & 10.13 & 36.84 & 35.13 & 36.84 & 10.13 \\
\hline $\begin{array}{c}0.25 \% \\
\text { BN(silane) }\end{array}$ & 10.99 & 3.11 & 514.8 & 10.44 & 39.11 & 36.46 & 39.11 & 9.27 \\
\hline $\begin{array}{c}0.25 \% \\
\text { BN(Acid) }\end{array}$ & 10.36 & 2.82 & 462.75 & 9.77 & 32.64 & 28.77 & 32.64 & 8.16 \\
\hline $\begin{array}{c}0.5 \% \\
\text { BN(silane) }\end{array}$ & 11.04 & 3.12 & 409.14 & 9.03 & 33.85 & 32.97 & 33.85 & 9.03 \\
\hline $\begin{array}{c}0.75 \% \\
\text { BN(silane) }\end{array}$ & 10.66 & 3.08 & 560.9 & 8.6 & 38.47 & 37.93 & 17.53 & 3.02 \\
\hline $\begin{array}{c}0.75 \% \\
\text { BN(Acid) }\end{array}$ & 11.17 & 2.97 & 482.22 & 9.29 & 36.89 & 36.88 & 36.89 & 9.27 \\
\hline
\end{tabular}


Figure 1. The comparison of the stretchability of neat epoxy and specimens managed $(0.25 \%)$

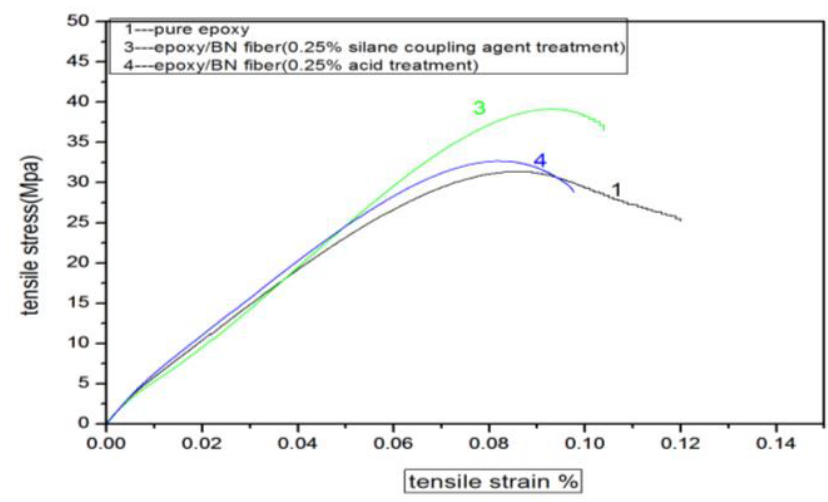

Fig.1 1showed the stress-strain curve of neat epoxy, composites added $\mathrm{BN}$ (acid) at the volume fraction of $0.25 \%$ and composites added BN (silane) at the volume fraction of $0.25 \%$. From the curve in Fig. 1 , by the same tensile stress, we could conclude that the tensile strain of composites added BN (silane) was enhanced slightly, but the tensile strain of composites added BN (acid) do not improved significantly.

Figure 2. The comparison of the stretchability of neat epoxy and specimens managed $(0.5 \%)$

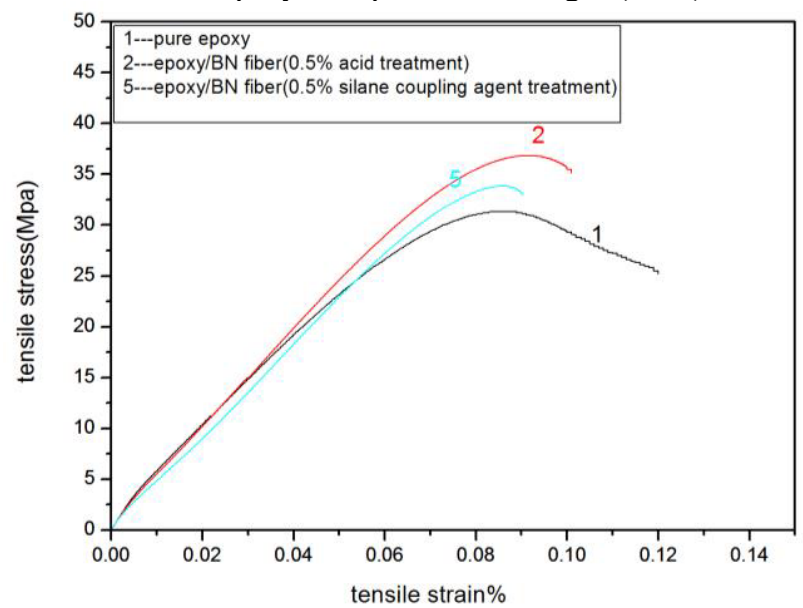

Fig.2showed the stress-strain curve of neat epoxy, composites added BN (acid) at the volume fraction of 0 . $5 \%$ and composites added $\mathrm{BN}$ (silane) at the volume fraction of $0.5 \%$ From the curve in Fig. 2 , by the same tensile stress, we could conclude that the tensile strain of composites added BN (acid) was enhanced slightly, but the tensile strain of composites added BN (silane) do not improved significantly.

Figure 3. The comparison of the stretchability of neat epoxy and specimens managed $(0.75 \%)$

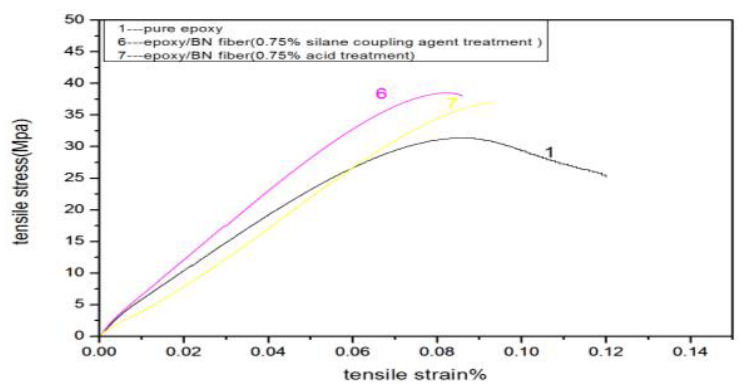

Fig.3showed the stress-strain curve of neat epoxy, composites added BN (acid) at the volume fraction of $0.75 \%$ and composites added BN (silane) at the volume fraction of $0.75 \%$ From the curve in Fig. 2 , by the same tensile stress, we could conclude that the tensile strain of composites added BN (silane) was enhanced slightly, but the tensile strain of composites added BN (acid) do not improved significantly.

Figure 4. The comparison of the stretchability of neat epoxy and specimens managed (silane)

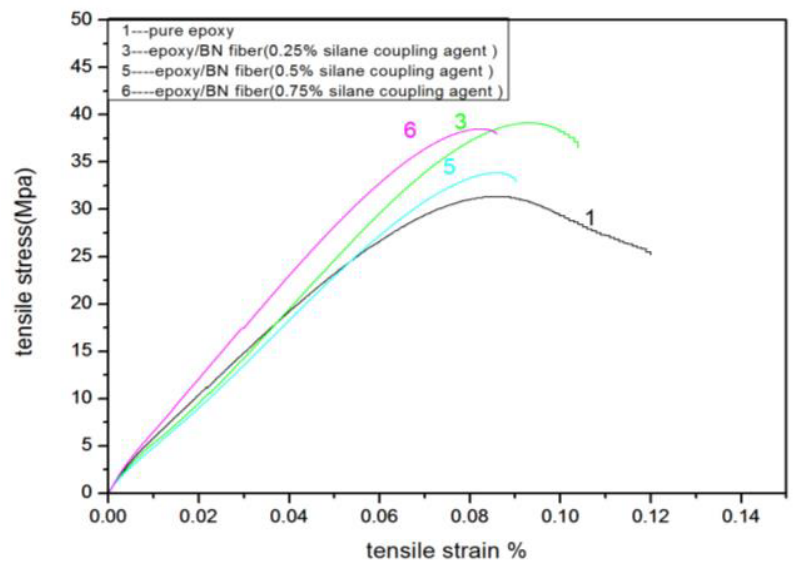

Fig.4showed the stress-strain curve of neat epoxy, composites added BN (silane) at the volume fraction of $0.25 \%$, composites added $\mathrm{BN}$ (silane) at the volume fraction of $0.5 \%$ and composites added $\mathrm{BN}$ (silane) at the volume fraction of $0.75 \%$. From the curve in Fig. 4 , by the same tensile stress, we could conclude that the tensile strain of composites added BN (silane) at the volume radio of $0.5 \%$ was enhanced slightly, but the tensile strain of composites added BN (silane) at the volume fraction of $0.25 \%$ and 0.75 do not improved significantly.

Fig. 5 showed the stress-strain curve of neat epoxy, composites added $\mathrm{BN}$ (acid) at the volume fraction of $0.25 \%$, composites added BN (acid) at the volume fraction of $0.5 \%$ and composites added $\mathrm{BN}$ (acid) at the volume fraction of $0.75 \%$. From the curve in Fig. 5 , by the same tensile stress, we could conclude that the tensile strain of composites added $\mathrm{BN}$ (acid) at the volume radio of $0.5 \%$ and $0.75 \%$ was enhanced slightly, but the tensile strain of composites added $\mathrm{BN}$ (acid) at the volume fraction of $0.25 \%$ do not improved significantly.

\subsection{Analysis of the tensile performance results}

From Table1 and Fig1 3, we could conclude that the tensile strength, elastic modulus, tensile fracture stress and tensile yield stress of the composites materials added silane coupling agent treatment or acidification treatment of BN exhibit enhanced obviously compared with neat epoxy, which proves that $\mathrm{BN}$ powers play a role in the composite materials, but the yield strain and the elongation at break decreased, what explain it is that adding $\mathrm{BN}$ powers developed the tensile fracture stress of composite materials. 
Figure 5. The comparison of the stretchability of neat epoxy and specimens managed (acid)

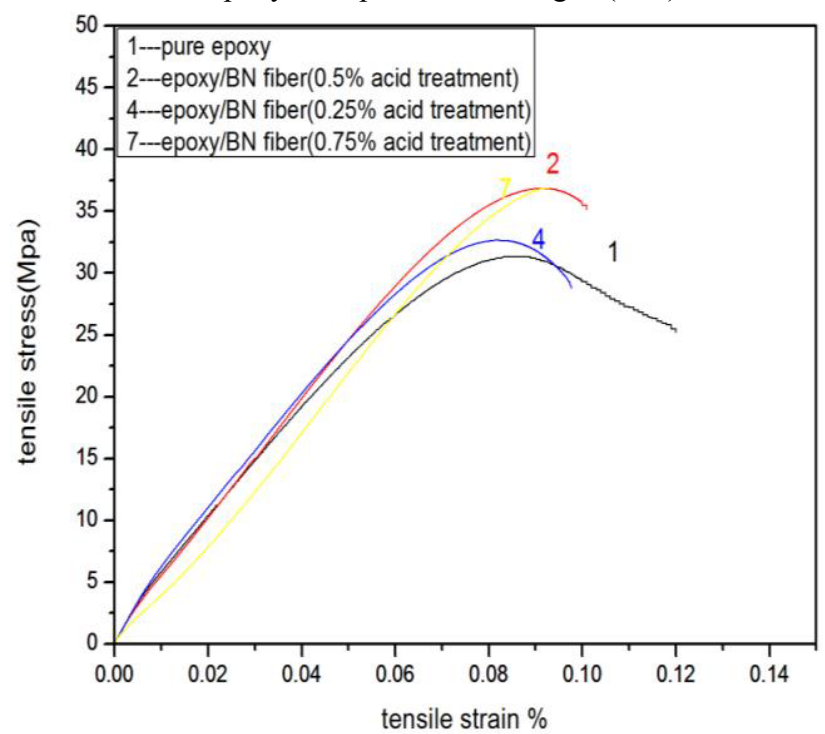

Fig.4 showed that the tensile strength of composite materials added BN (silane) is higher than the tensile strength of composite material added $\mathrm{BN}$ (acid) at the same volume fraction, because the structure of BN was destroyed because of acidification, which makes it difficult to perform the net structure in the composites.

Fig. 5 showed that the tensile strength of composite material increase continuously and the tensile yield stress of composite material decrease continuously with the growth of BN (acid or silane), the reason is that the net structure changes more and more obviously and the scope of reach of the net becomes bigger and bigger, which makes the tensile fracture stress gets higher and higher.

\subsection{Reasons and discussion of thermal conductivity of composite material}

From Table3, the thermal conductivity of composites added $\mathrm{BN}$ (acid or silane) exhibit enhanced obviously compared with neat epoxy, which proves that $\mathrm{BN}$ powers play a role in the composite materials. But the thermal conductivity of composites add BN (acid or silane) at the volume fraction of $0.25 \%$ decrease slightly compared with neat epoxy, the reason is that adding $\mathrm{BN}$ powers cuts the original chain of thermal conductivity, which not only do not improve the ability of heat conduction but also makes it lower.

The thermal conductivity of composites added BN (silane) is lower than the composites added BN (acid) at the same volume radio of BN powers, the reason is that the acidification enhances the interaction between fillers of $\mathrm{BN}$ and matrix, which makes it easy to perform net structure, the net could be used to the faster path to complete the heat dispersion, which develops the thermal conductivity of composites.

The coefficient of thermal conductivity of composite material increase continuously and the tensile yield stress of composite material decrease continuously with the growth of BN (acid or silane), because the area of the net in the composites becomes bigger and bigger, the thermal conductive path is more and more intensive, which is more conducive for the heat condution.

Table 2. The tensile data of the specimens.

\begin{tabular}{|c|c|c|c|c|c|}
\hline $\begin{array}{c}\text { serial } \\
\text { number }\end{array}$ & type & $\begin{array}{c}\text { parameter } \\
(\mathbf{m m})\end{array}$ & \multicolumn{3}{|c|}{ data } \\
\hline \multirow{2}{*}{1} & \multirow{2}{*}{ neat } & $\begin{array}{l}\text { width } \\
\text { (W) }\end{array}$ & 10.86 & 10.83 & 10.72 \\
\hline & & $\begin{array}{l}\text { thickness } \\
\text { (d) }\end{array}$ & 3.18 & 3.21 & 3.2 \\
\hline \multirow{2}{*}{2} & \multirow{2}{*}{$\begin{array}{c}0.5 \% \\
\text { BN(Acid) }\end{array}$} & $\begin{array}{l}\text { width } \\
\text { (W) }\end{array}$ & 10.77 & 10.76 & 10.88 \\
\hline & & $\begin{array}{l}\text { thickness } \\
\text { (d) }\end{array}$ & 3.16 & 3.18 & 3.09 \\
\hline \multirow{2}{*}{3} & \multirow{2}{*}{$\begin{array}{c}0.25 \% \\
\text { BN(silane) }\end{array}$} & $\begin{array}{l}\text { width } \\
\text { (W) }\end{array}$ & 11.05 & 10.99 & 10.97 \\
\hline & & $\begin{array}{l}\text { thickness } \\
\text { (d) }\end{array}$ & 3.11 & 3.13 & 3.22 \\
\hline \multirow{2}{*}{4} & \multirow{2}{*}{$\begin{array}{c}0.25 \% \\
\text { BN(Acid) }\end{array}$} & $\begin{array}{l}\text { width } \\
\text { (W) }\end{array}$ & 10.36 & 10.55 & 10.4 \\
\hline & & $\begin{array}{l}\text { thickness } \\
\text { (d) }\end{array}$ & 2.87 & 2.82 & 2.88 \\
\hline \multirow{2}{*}{5} & \multirow{2}{*}{$\begin{array}{c}0.5 \% \\
\text { BN(silane) }\end{array}$} & $\begin{array}{l}\text { width } \\
\text { (W) }\end{array}$ & 11.06 & 11.04 & 11.02 \\
\hline & & $\begin{array}{l}\text { thickness } \\
\text { (d) }\end{array}$ & 3.11 & 3.12 & 3.18 \\
\hline \multirow{2}{*}{6} & \multirow{2}{*}{$\begin{array}{c}0.75 \% \\
\text { BN(silane) }\end{array}$} & $\begin{array}{l}\text { width } \\
\text { (W) }\end{array}$ & 10.63 & 10.69 & 10.66 \\
\hline & & $\begin{array}{l}\text { thickness } \\
\text { (d) }\end{array}$ & 3.09 & 3.08 & 3.2 \\
\hline \multirow{2}{*}{7} & \multirow{2}{*}{$\begin{array}{c}0.75 \% \\
\text { BN(Acid) }\end{array}$} & $\begin{array}{l}\text { width } \\
\text { (W) }\end{array}$ & 11.17 & 11.3 & 11.24 \\
\hline & & $\begin{array}{l}\text { thickness } \\
\text { (d) }\end{array}$ & 2.96 & 3 & 2.97 \\
\hline
\end{tabular}

Figure 6. Coefficient of thermal conductivity contrast before and after processing

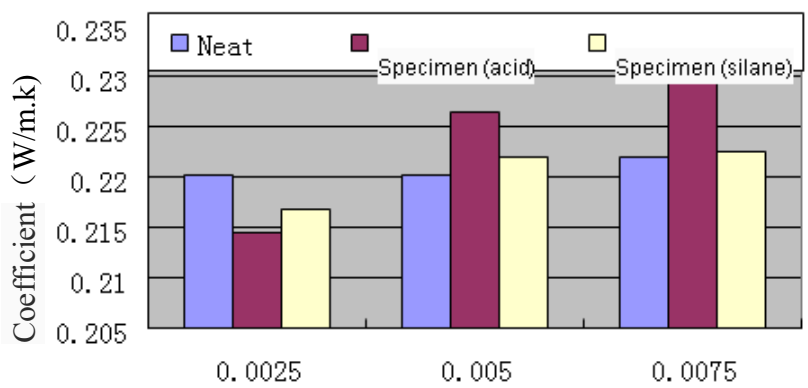


Table 3. The thermal conductivity of epoxy/BN composites.

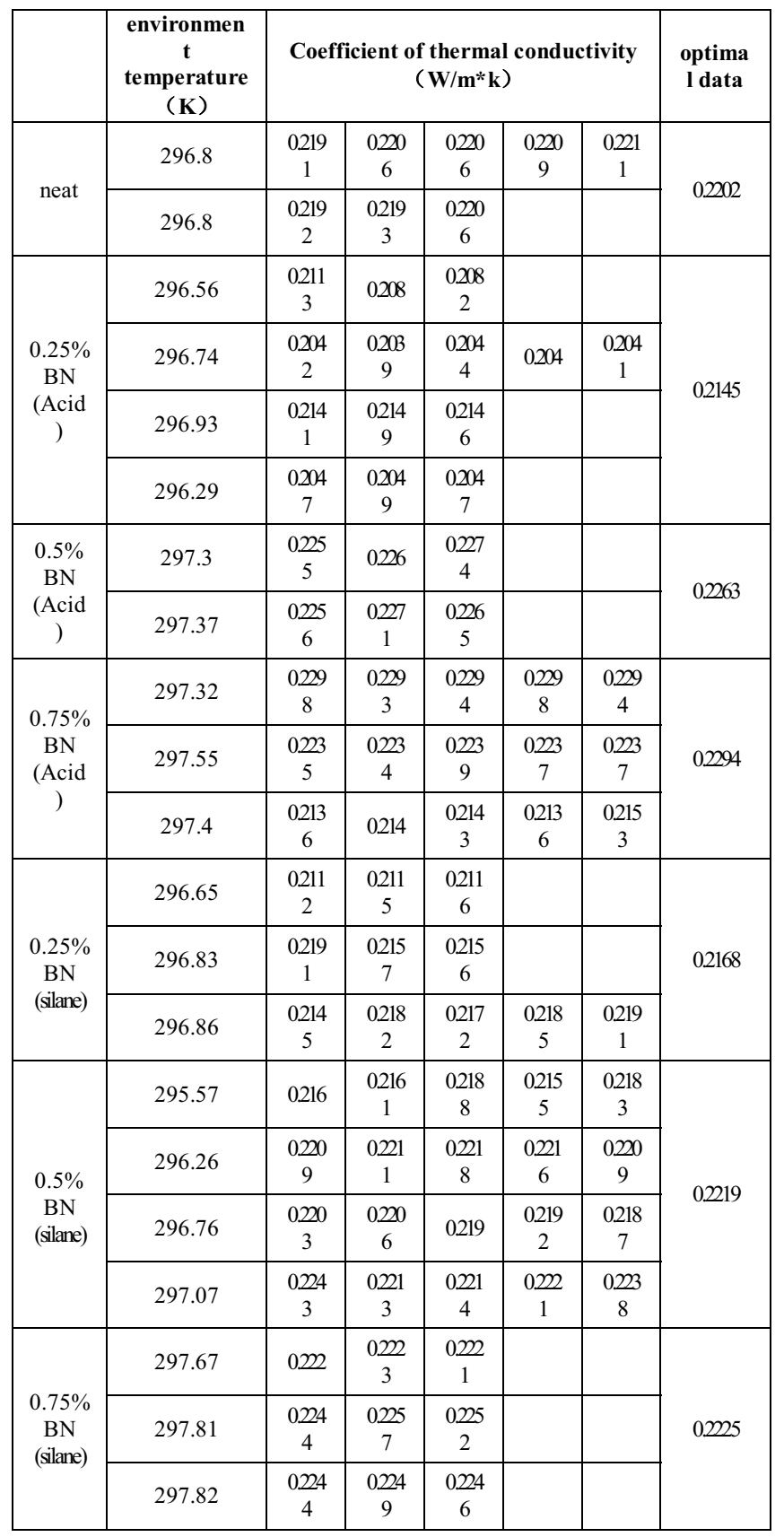

\section{Conclusions}

The tensile strength, elastic modulus, tensile fracture stress and tensile yield stress of the composites materials added silane coupling agent treatment or acidification treatment of $\mathrm{BN}$ exhibit enhanced obviously compared with neat epoxy, which proves that $\mathrm{BN}$ powers play a role in the composite materials, but the yield strain and the elongation at break decreased, and the thermal conductivity of composites added $\mathrm{BN}$ (acid) and $\mathrm{BN}$ (silane) at the same volume radio of 0.25 also exhibit the trend of decline, what explain it is that the volume fraction of $\mathrm{BN}$ is too little, $\mathrm{BN}$ added to the composites could not only not form a network structure to provide channels for thermal diffusion but also destroyed the original heat conduction path.
At the same volume fraction, fillers treated with a silane coupling agent provide composites with lower thermal conductivity and higher tensile strength compared with the ones treated with acid.

With the increase of the volume fraction of fillers, the thermal conductivity and the tensile strength of the composites show the tendency of increase, but the tensile yield stress of composites exhibit significant reduction.

\section{References}

1. Yuanxin Zhou, Farhana Pervin, Lance Lewis, Shaik Jeelani, Experimental study on the thermal and mechanical properties of multi-walled carbon nanotube-reinforced epoxy. Material Science and Engineering A 452-453,657-664 (2007)

2. Jinhong Yu, Xingyi Huang*, Chao Wu, Xinfeng Wu, Genlin Wang, Pingkai Jiang*, Interfacial modification of boron nitride nanoplatelets for epoxy composites with improved thermal properties. Polymer 53,471-480 (2012)

3. Kickelbick G. Prog Polym Sci 28(1):83-114 (2003).

4. Rozenberg BA, Tenne R. Prog Polym Sci 33(1):40112 (2008).

5. Pyun J, Kowalewski T, Matyjaszewski K. Macromol Rapid Commun 24(18):1043-59 (2003)

6. Chan CM, Wu J, Li JX, Cheung YK. Polymer 43(10):2981-92 (2002)

7. Maclachlan MJ, Manners L, Ozin GA. Adv Mater 12(9):675-81 (2000)

8. R. Bagheri, R.A. Pearson, Polymer 41 269-276 (2001)

9. T. Kawaguchi, R. A. Pearson, Polymer 44 4239-4247 (2003)

10. H. Mahfuz, A. Adnan, V. K. Rangari, S. Jeelani, B. Z. Jang, Compos. Part A : Appl. Sci. Manuf. 35 519527 (2004)

11. M. F. Evora, A. Shukla, Mater. Sci. Eng. A 361 358-366 (2003)

12. R. Rodgers, H. Mahfuz, V. Rangari, N. Chisholm, S. Jeelani, Macromol. Mater. Eng. 290 (5) 423-429 (2005)

13. P. Farhana, Y.X.Zhou, V. Rangari, S. Jeelani, Mater. Sci. Eng. A 405 (1-2) 246-253 (2005)

14. Y. H. Liao, M.T. Olivier, Z. Y. Liang, C. Zhang, B. Wang, Mater. Sci. Eng. A 385 175-181 (2004)

15. Golberg D, Bando Y, Huang Y, Terao T, Mitome M, Tang C, et al. ACS Nano 4(6):2979-93 (2010)

16. Golberg D, Bando Y, Tang CC, Zhi CY. Adv Mater 19 (18):2413-32 (2007)

17. Barus S, Zanetti M, Lazzari M, Costa L. Polymer 50(12):2595-600 (2009)

18. Sanchez C, Julian B, Belleville P, Popall M. J Mater Chem 15(35-36):3559-92 (2005)

19. Herman Teo HK, Toh CL, Lu X. Polymer 52(9):1975-82 (2011)

20. Terao T, Zhi C, Bando Y, Mitome M, Tang C, Golberg D. J Phys Chem C 114(10):4340-4 (2010)

21. Zhi C, Bando Y, Tang C, Kuwahara H, Golberg D. Adv Mater 21(28):2889-93 (2009) 